\title{
Acides gras polyinsaturés $n-3$, neurotransmission et fonctions cognitives
}

Oléagineux, Corps Gras, Lipides. Volume 7, Numéro 1, 68-73, Janvier - Février 2000, Dossier : actes des Journées Chevreul "Corps gras, nutrition et santé, questions d'actualité" (Bordeaux, Pessac)

Auteur(s) : Sylvie CHALON, Inserm U316, Laboratoire de biophysique médicale et pharmaceutique, Faculté de pharmacie, 31, avenue Monge, 37200 Tours, France.

Résumé : Les acides gras polyinsaturés (AGPI) incorporés dans les phospholipides membranaires représentent une proportion importante (environ $20 \%$ ) de la matière sèche cérébrale. Ils sont apportés par l'alimentation sous forme de précurseurs (acide a-linolénique pour la série $n-3$, acide linoléique pour la série $\mathrm{n}-6$ ) ou de dérivés à longues chaînes (notamment l'acide docosahexaénoïque, ou 22:6n-3 et l'acide arachidonique, ou 20:4n-6). Des données récentes indiquent que le statut en AGPI constitue l'un des facteurs environnementaux capables d'affecter le fonctionnement du système nerveux central (SNC). Les résultats principaux sont, d'une part, la mise en évidence d'anomalies comportementales chez des animaux recevant des régimes déséquilibrés en AGPI et, d'autre part, la détection de taux anormaux d'AGPI chez des humains souffrant de maladies du SNC. $\mathrm{Au}$ cours de ces dernières années, nous avons apporté des arguments établissant que ces dysfonctionnements peuvent être reliés à des altérations des processus de neurotransmission induites par le déséquilibre en AGPI.

Summary : Polyunsaturated fatty acids are main components of cerebral membranes. Recent data demonstrate that they are involved in the functioning of the central nervous system. Peripheral measurements of PUFA in plasma or red blood cells show that the amount of $n-6$ and mainly $n-3$ PUFA can be very variable among healthy humans. These PUFA levels can be reduced in several neurological or psychiatric disorders, such as schizophrenia, depression, and attention deficithyperactivity disorders. These decreases are stronger in red blood cells than in plasma, and mainly affect the levels of docosahexaenoic acid. The cause of this deficit is still unclear. However, it can be noted that these three diseases could be associated with abnormalities of monoaminergic (dopaminergic, serotoninergic) neurotransmission. We have recently demonstrated that these neurotransmission processes are altered in a rat model of chronic n-3 PUFA deficiency. The mesocorticolimbic dopaminergic system, which is involved in attentional and motivational behaviour, seems to be imbalanced with a pronounced deficit in the vesicle storage pool of dopamine. The overall clinical and animal findings suggest that relationships may occur between $n-3$ PUFA status, cerebral neurochemistry, and abnormal behaviour.

Keywords : dopamine, n-3 PUFA, serotonin, schizophrenia. 


\section{ARTICLE}

\section{Acides gras polyinsaturés et maladies du SNC chez l'homme : données cliniques}

Plusieurs groupes se sont intéressés au statut en AGPI de sujets souffrant de maladies neurologiques ou psychiatriques. L'une des difficultés majeures de l'interprétation de ces données épidémiologiques est la comparaison des résultats obtenus chez les sujets malades avec ceux émanant d'une population dite normale. En effet, il semble que les taux d'AGPI n- 6 et surtout d'AGPI n-3 puissent varier de manière importante en fonction de différents groupes humains. Une étude récente [1] indique que la fraction d'AGPI $n-3$ contenue dans les acides gras totaux des phospholipides plasmatiques peut varier d'un facteur 3 entre différentes populations d'adultes (tableau 1). Il est important de constater dans cette étude que le pourcentage d'AGPI n-3 est beaucoup plus hétérogène que celui des AGPI n-6 (variations d'un facteur 1,5 dans cette même étude).

En considérant comme sujets témoins un groupe de 100 adultes américains du Minnesota consommant un régime omnivore, Holman et al. [1] décrivent des diminutions légères des taux d'AGPI n-6 (autour de 10 à $15 \%$ ) et plus importantes d'AGPI n-3 (autour de 20 à $35 \%$ ) dans diverses maladies du SNC telles que l'anorexie nerveuse, la maladie de Huntington, la sclérose multiple, la rétinite pigmentaire (tableau 2). II faut cependant noter que le nombre de sujets étudiés est assez faible (de 8 à 28 selon la maladie).

Nous nous sommes plus particulièrement intéressés à des maladies psychiatriques pour lesquelles on dispose à la fois de données montrant un déficit en AGPI, et d'arguments en faveur d'une implication des systèmes de neurotransmission mono-aminergique (en particulier dopaminergique et sérotoninergique) : la schizophrénie, la dépression, les troubles de l'attention chez l'enfant (ADHD). Dans ces maladies, des anomalies du statut en AGPI ont été détectées au niveau périphérique (plasma ou érythrocytes), ce qui représente un reflet indirect de la composition en acides gras des membranes neuronales.

\section{La schizophrénie}

La schizophrénie est une maladie mentale sévère qui touche environ $1 \%$ de la population, et dans laquelle on trouve des symptômes dits positifs et/ou négatifs [2]. Les données les plus récentes définissent la schizophrénie comme une maladie neurodéveloppementale, c'est-à-dire débutant pendant la période de mise en place des fonctions cérébrales, bien que les premiers symptômes n'apparaissent en général que chez l'adolescent ou le jeune adulte.

Les altérations physiologiques accompagnant cette maladie sont multiples. En effet, des anomalies biochimiques ou génétiques ont pu être détectées au niveau périphérique (études plasmatiques ou urinaires) ; de plus, des anomalies anatomiques, métaboliques ou neurochimiques ont été mises en évidence au niveau du SNC (en particulier grâce à des études d'imagerie cérébrale). Des dysfonctionnements des systèmes dopaminergiques pourraient jouer un rôle prépondérant dans les manifestations de la maladie [3]. Les causes de la schizophrénie sont probablement diverses, mais il semble que la présence d'anomalies génétiques associées à des facteurs environnementaux particuliers (par exemple l'infection in utero) pourrait apparaître comme facteur déclenchant. 
Les travaux d'Horrobin ont été les premiers à relier schizophrénie et anomalies du statut en AGPI. Une diminution du taux de certains acides gras essentiels (AGE), en particulier l'acide linoléique, a été décrite dans le plasma de sujets schizophrènes comparés à des témoins [4,5]. Cependant, les données les plus nombreuses et les plus convergentes concernent les dosages au niveau des membranes érythrocytaires (tableau 3). En effet, plusieurs études ont mis en évidence l'existence de deux populations de schizophrènes : I'une dont les taux d'AGE sont identiques à ceux des sujets témoins, l'autre présentant des diminutions significatives des taux de certains AGPI n-6 et n-3, en particulier l'acide arachidonique et l'acide docosahexaénoïque [6-8]. De plus, le déficit érythrocytaire en AGPI semble relié aux symptômes négatifs des sujets schizophrènes, tandis que des taux normaux sont retrouvés chez les sujets de type positif.

Plusieurs mécanismes peuvent être à l'origine de ce déficit membranaire en AGPI. L'hypothèse émise et défendue par Horrobin est que l'activité de la phospholipase A2 est augmentée chez certains schizophrènes, induisant ainsi une extraction accrue de l'acide arachidonique et de l'acide docosahexaénoïque membranaires [9-11]. Un argument en faveur d'un effet du déficit en AGE sur la schizophrénie est que la supplémentation alimentaire en AGPI peut améliorer les symptômes de la maladie $[12,13]$. Ainsi, ce type de supplémentation associé à des traitements pharmacologiques pourrait apparaître comme une perspective intéressante qui demanderait à être évaluée par d'autres groupes travaillant sur la schizophrénie.

\section{La dépression}

La dépression est une maladie complexe qui implique notamment les systèmes de neurotransmission sérotoninergiques, et plus particulièrement les récepteurs et le transporteur (site d'action d'antidépresseurs) de la sérotonine [14].

Plusieurs études ont mis en évidence un déficit plasmatique et/ou érythrocytaire en AGPI au cours de la dépression [15-18]. Par exemple, des diminutions significatives des taux d'AGPI n-6 et surtout d'AGPI n-3 (diminution d'environ $45 \%$ des acides alpha-linolénique et docosahexaénoïque) ont été mesurées dans les érythrocytes de patients déprimés, conduisant ainsi à une augmentation globale du rapport des AGPI n-6/AGPI n-3 (tableau 4). Cependant, aucune hypothèse n'est encore avancée quant à la survenue de ces anomalies.

Les troubles de l'attention (ADHD ou Attention Deficit and Hyperactivity Disorders)

Les signes majeurs de I'ADHD, qui touche préférentiellement les garçons, sont un manque d'attention, une impulsivité accrue et une hyperactivité [19]. Décrite comme la maladie psychiatrique la plus répandue chez l'enfant, I'ADHD semble associé à certaines anomalies de la neurotransmission dopaminergique [20].

Les premiers travaux reliant ADHD et AGPI ont comparé les taux plasmatiques d'AGPI chez 48 enfants non traités souffrant d'hyperactivité et des sujets témoins appariés en âge [21]. Ils ont mis en évidence une diminution des concentrations d'acide docosahexaénoïque, d'acide arachidonique et d'acide dihomogammalinolénique (20:3 n-6). 
Des études plus récentes ont confirmé le déficit plasmatique en AGPI chez des sujets atteints et observé le même résultat au niveau érythrocytaire (tableau 5) [22]. Les mécanismes qui pourraient rendre compte des anomalies du statut en AGPI au cours de I'ADHD sont encore inconnus.

Ainsi, un certain nombre de données cliniques montrent que des déficits périphériques (plasmatiques, érythrocytaires) en AGPI n-6 et surtout en AGPI n-3 peuvent accompagner plusieurs maladies du SNC dont la schizophrénie, la dépression et I'ADHD. L'origine de ces anomalies reste à déterminer. Plusieurs hypothèses peuvent être proposées parmi lesquelles un déficit d'apport alimentaire ou d'absorption des AGPI, une mauvaise incorporation membranaire ou une extraction accrue (hypothèse d'Horrobin) de ces AGPI. Par ailleurs, il reste à montrer que les anomalies périphériques de la composition en AGPI reflètent bien les mêmes anomalies au niveau des membranes neuronales. Les mécanismes pouvant expliquer les effets du déficit en AGPI sur les maladies psychiatriques que nous avons évoquées restent à élucider. Dans ces trois maladies, des altérations des systèmes de neurotransmission mono-aminergique, en particulier dopaminergique et/ou sérotoninergique, qui ont un rôle majeur dans la modulation des fonctions cérébrales, ont été mises en évidence. De plus, notre équipe a apporté un certain nombre d'arguments, chez l'animal, qui démontrent des effets du statut en AGPI sur la neurotransmission dopaminergique. Il est donc possible chez l'humain que certains troubles comportementaux soient liés à des altérations des processus de neurotransmission monoaminergique, ces altérations pouvant être induites ou plus vraisemblablement aggravées par un déséquilibre en AGPI. Une telle hypothèse peut être étudiée en utilisant des modèles animaux dans lesquels les relations entre manipulations alimentaires, paramètres neurochimiques et paramètres comportementaux peuvent être déterminées, puis comparées aux études cliniques.

\section{Acides gras polyinsaturés et neurotransmission : études chez l'animal}

Le modèle de la déficience alimentaire chronique en acide alpha-linolénique chez le rat nous a permis de mettre en évidence, pour la première fois, des effets neurochimiques. Ce type de déficience entraîne chez le rat et le singe une altération de la vision (électrorétinogramme, acuité visuelle) et des perturbations comportementales [23, 24]. Ces dernières consistent notamment en une réduction de l'activité exploratoire, une altération du processus d'extinction et une diminution des capacités d'apprentissage discriminatif [25]. Ces anomalies comportementales correspondent à celles décrites lors de lésions expérimentales des systèmes dopaminergiques méso-cortical et mésolimbique [26-28]. Nous avons alors émis l'hypothèse que ces systèmes de neurotransmission pouvaient être impliqués dans les perturbations comportementales induites par la déficience en AGPI n-3.

En utilisant le modèle expérimental du rat soumis à différentes manipulations alimentaires, nous avons en effet montré que des apports déséquilibrés en AGPI n-3 (déficience chronique en acide alpha-linolénique, surcharge chronique en AGPI à longues chaînes) induisent des effets sur plusieurs paramètres de la neurotransmission dopaminergique.

Dans le modèle de déficience, les animaux reçoivent depuis plusieurs générations une ration lipidique composée exclusivement d'huile d'arachide dépourvue d'AGPI n-3. Ces animaux sont comparés à des témoins recevant un mélange d'huile d'arachide et d'huile de colza riche en acide 
alpha-linolénique. Dans le régime déficient, le rapport AGPI n-6 sur AGPI n-3 est supérieur à 200, alors qu'il est égal à 6,2 dans le régime témoin [29]. La composition en acides gras des phospholipides membranaires cérébraux se caractérise par une diminution d'environ $68 \%$ des AGPI n-3, compensée par une augmentation de même ordre des AGPI n-6 [23, 29, 30].

Chez ces animaux, nous avons mis en évidence des anomalies du métabolisme dopaminergique et de certaines cibles moléculaires de la dopamine dans deux régions cérébrales spécifiques, le cortex frontal et le noyau accumbens, où sont localisées respectivement les terminaisons méso-corticales et méso-limbiques [29-33]. Le métabolisme de la dopamine a été évalué par la méthode de microdialyse. Cette méthode permet l'étude dynamique de la libération basale ou stimulée de la dopamine et de ses métabolites chez l'animal vivant. Les cibles moléculaires de la dopamine (récepteurs, transporteurs membranaires et vésiculaires) ont été étudiées à l'aide de radioligands spécifiques, par méthode d'autoradiographie quantitative. La mise en œuvre de ces différentes méthodes nous permet de comparer le fonctionnement de paramètres essentiels de la neurotransmission dopaminergique dans des lots d'animaux soumis à différentes manipulations alimentaires.

Les anomalies métaboliques principales de la dopamine chez les animaux chroniquement déficients en AGPI n-3 sont :

- une diminution de la libération de dopamine basale associée à une augmentation de la libération des métabolites (Dopac, HVA) dans le cortex frontal (figure 1);

- une augmentation de la libération de dopamine basale associée à une diminution de la libération des métabolites dans le noyau accumbens (figure 2);

- une diminution du pool de réserve de la dopamine (contenu dans les vésicules de stockage) dans les deux régions [31] ;

- une augmentation de l'activité tyrosine hydroxylase (enzyme limitante de la synthèse de dopamine) dans les corps cellulaires des neurones dopaminergiques localisés au niveau de l'aire tegmentale ventrale [32].

Les anomalies des cibles moléculaires de la dopamine concernent, d'une part, les récepteurs dopaminergiques D2 (diminution dans le cortex frontal, augmentation dans le noyau accumbens), et d'autre part, le transporteur vésiculaire des mono-amines (diminution dans les deux zones cérébrales).

L'ensemble de ces résultats montre que la déficience chronique en AGPI n-3 affecte les systèmes de neurotransmission dopaminergique méso-cortical et méso-limbique, et nous a permis de proposer un mécanisme pouvant rendre compte des anomalies détectées (figure 3).

* La déficience en AGPI n-3 induit un déficit (diminution du nombre et/ou de la fonctionnalité) des vésicules de stockage contenant la dopamine dans le cortex frontal et le noyau accumbens. Ce déficit pourrait être dû à des anomalies de la structure membranaire des vésicules induites par la modification de sa composition en AGPI. Ces anomalies pourraient entraîner des perturbations 
physico-chimiques telles qu'une fragilité des membranes ou une altération de leur fluidité pouvant affecter le processus de mise en réserve de la dopamine.

* La diminution de la mise en réserve de la dopamine dans les vésicules pourrait perturber l'ensemble du métabolisme de ce neurotransmetteur dans le cortex frontal (diminution du taux de dopamine, augmentation du taux de ses métabolites) et dans le noyau accumbens. Dans cette région limbique, l'augmentation du taux de dopamine pourrait être due, d'une part, à la levée de l'inhibition exercée par le cortex frontal et, d'autre part, à l'augmentation de la synthèse (augmentation de l'activité tyrosine hydroxylase dans l'aire tegmentale ventrale).

* Les anomalies du taux de récepteurs de la dopamine pourraient être liées aux anomalies de la structure des membranes dans lesquelles ils sont enchâssés. Cependant, on ne peut exclure un effet direct des AGPI ou de leurs dérivés au niveau du génome, conduisant à une modification de l'expression de ces récepteurs.

Étant donné le rôle joué par les systèmes dopaminergiques méso-cortical et méso-limbique dans les processus comportementaux, les anomalies neurochimiques que nous avons mises en évidence peuvent expliquer la réactivité comportementale accrue et les difficultés d'apprentissage décrites dans ce type de déficience $[24,34,35]$.

Ainsi, ces travaux constituent les premiers arguments expérimentaux établissant des modifications de processus neurochimiques induites par un déséquilibre alimentaire en AGPI. La juxtaposition de ces résultats avec certaines données obtenues chez l'homme indique que ce déséquilibre pourrait participer à des manifestations cliniques de maladies cérébrales impliquant les systèmes de neurotransmission mono-aminergique. Ceci ouvre la voie à de nombreuses perspectives en termes de prévention et de traitements complémentaires des maladies du SNC, en considérant qu'une amélioration du statut nutritionnel pourrait améliorer la résistance aux troubles du fonctionnement cérébral.

\section{REFERENCES}

1. HOLMAN RT (1997). omega3 and omega6 essential fatty acid status in human health and disease. In : YEHUDA S, MOSTOFSKY DI, eds. Handbook of essential fatty acid biology : biochemistry, physiology, and behavioral neurobiology. Totowa, NJ : Humana Press Inc : 139-82.

2. CARPENTER WT, HEINRICHS DW, WAGMAN AMI (1988). Deficit and nondeficit forms of schizophrenia : the concept. Am J Psychiat, 145 : 578-83.

3. DAVIS KL, KAHN RS, KO G, DAVIDSON M (1991). Dopamine in schizophrenia : a review and reconceptualization. Am J Psychiat, 148 : 1474-86.

4. HORROBIN DF, MANKU MS, MORSE-FISHER N, VADDADI KS, COURTNEY P, GLEN AIM, GLEN E, SPELLMAN M, BATES C (1989). Essential fatty acids in plasma phospholipids in schizophrenics. Biol Psychiat, 25 : 562-8. 
5. KAIYA H, HORROBIN DF, MANKU MS, MORSE-FISHER N (1991). Essential and other fatty acids in schizophrenic individuals from Japan. Biol Psychiat, 30 : 357-62.

6. GLEN AIM, GLEN EMT, HORROBIN DF, VADDADI KS, SPELLMAN M, MORSE-FISHER N, ELLS K, SHINNER FS (1994). A red cell membrane abnormality in a subgroup of schizophrenic patients : evidence of two diseases. Schizophr Res, 12 : 53-61.

7. PEET M, LAUGHARNE JD, HORROBIN DF, REYNOLDS GP (1994). Arachidonic acid : a common link in the biology of schizophrenia. Arch Gen Psychiat, 51 : 665-6.

8. PEET M, LAUGHARNE JD, RANGARAJAN N, HORROBIN D, REYNOLDS G (1995). Depleted red cell membrane essential fatty acids in drug-treated schizophrenic patients. J Psychiat Res, 29 : 227-32.

9. HORROBIN DF, GLEN AIM, VADDADI K (1994). The membrane hypothesis of schizophrenia. Schizophr Res, 13 : 195-207.

10. HORROBIN DF (1997). Fatty acids, phospholipids, and schizophrenia. In : YEHUDA S, MOSTOFSKY DI, eds. Handbook of essential fatty acid biology : biochemistry, physiology, and behavioral neurobiology. Totowa, NJ : Humana Press Inc : 245-56.

11. HORROBIN DF (1998). The membrane phospholipid hypothesis as a biochemical basis for the neurodevelopmental concept of schizophrenia. Schizophr Res, 30 : 193-208.

12. PEET M, LAUGHARNE JD, MELLOR J, RAMCHAND CN (1996). Essential fatty acid deficiency in erythrocyte membranes from chronic schizophrenic patients, and the clinical effects of dietary supplementation. Prostaglandins Leukot Essent Fatty Acid, 55 : 71-5.

13. MELLOR J, LAUGHARNE JD, PEET M (1995). Schizophrenic symptom and dietary intake of $n-3$ fatty acids. Schizophr Res, $18:$ 85-6.

14. MELTZER HY (1990). Role of serotonin in depression. Ann NY Acad Sci, 600 : 486-99.

15. HIBBELN JR, SALEM N (1995). Dietary polyunsaturated fatty acids and depression : when cholesterol does not satisty. Am J Clin Nutr, 62 : 1-9.

16. ADAMS PB, LAWSON S, SANIGORSKI A, SINCLAIR AJ (1996). Arachidonic acid to eicosapentaenoic acid ratio in blood correlates positively with clinical symptoms of depression. Lipids, 31 : S157-61.

17. PEET M, MURPHY B, SHAY J, HORROBIN DF (1998). Depletion of omega-3 fatty acid levels in red blood cell membranes of depressive patients. Biol Psychiat, $43:$ 315-9.

18. EDWARDS R, PEET M, SHAY J, HORROBIN D (1998). Omega-3 polyunsaturated fatty acid levels in the diet and in red blood cell membranes of depressed patients. J Affect Disorders, $48: 149-55$.

19. AMERICAN PSYCHIATRIC ASSOCIATION (1994). Diagnostic and Statistical Manual for Mental Disorders, 4th ed. Washington : DC, American Psychiatric Press.

20. CASTELLANOS FX (1997). Toward a pathophysiology of attention-deficit/hyperactivity disorder. Clin Pediatr, $36:$ 381-93. 
21. MITCHELL EA, AMAN MG, TURBOTT SH, MANKU M (1987). Clinical characteristics and serum essential fatty acid levels in hyperactive children. Clin Pediatr, 26 : 406-11.

22. STEVENS LJ, ZENTALL SS, DECK JL, ABATE ML, WATKINS BA, LIPP SR, BURGESS JR (1995). Essential fatty acid metabolism in boys with attention-deficit hyperactivity disorder. Am J Clin Nutr, 62 : 761-8.

23. BOURRE JM, FRANÇOIS M, YOUYOU A, DUMONT O, PICIOTTI M, PASCAL G, DURAND G (1989). The effects of dietary alpha-linolenic acid on the composition of nerve membranes, enzymatic activity, amplitude of electrophysiological parameters, resistance to poisons and performance of learning task in rat. J Nutr, $119: 1880-92$.

24. NEURINGER M, ANDERSON GJ, CONNOR WE (1988). The essentiality of $n-3$ fatty acids for the development and function of the retina and brain. Ann Rev Nutr, 8 : 517-41.

25. REISBICK S, NEURINGER M (1997). Omega-3 fatty acid deficiency and behaviour : a critical review and directions for future research. In : YEHUDA S, MOSTOFSKY DI, eds. Handbook of essential fatty acid biology : biochemistry, physiology, and behavioral neurobiology. Totowa, NJ : Humana Press Inc : 397-425.

26. SIMON H, SCATTON B, LE MOAL M (1980). Dopaminergic A10 neurons are involved in cognitive functions. Nature, $286: 150-1$.

27. TAGHZOUTI K, SIMON H, LOUILOT A, HERMAN JP, LE MOAL M (1985). Behavioral study after local injection of 6-hydroxydopamine in the nucleus accumbens of the rat. Brain Res, 344 : 9-20.

28. LE MOAL M, SIMON R (1991). Mesocorticolimbic dopaminergic networks : functional and regulatory roles. Physiol Rev, $71: 155-234$.

29. DELION S, CHALON S, HERAULT J, GUILLOTEAU D, BESNARD JC, DURAND G (1994). Chronic dietary alpha-linolenic acid deficiency alters dopaminergic and serotoninergic neurotransmission in rats. $J$ Nutr, 124 : 2466-76.

30. DELION S, CHALON S, GUILLOTEAU D, BESNARD JC, DURAND G (1996). alpha-linolenic acid dietary deficiency alters age-related changes of dopaminergic and serotoninergic neurotransmission in the rat frontal cortex. J Neurochem, 66 : 1582-91.

31. ZIMMER L, HEMBERT S, DURAND G, BRETON P, GUILLOTEAU D, BESNARD JC, CHALON S (1998). Chronic $n-3$ polyunsaturated fatty acid diet-deficiency acts on dopamine metabolism in the rat frontal cortex : a microdialysis study. Neurosci Lett, $240: 177-81$.

32. ZIMMER L, BRETON P, DURAND G, GUILLOTEAU D, BESNARD JC, CHALON S (1999). Prominent role of $\mathrm{n}-3$ polyunsaturated fatty acids in cortical dopamine metabolism. Nutritional Neuroscience, 2 : 257-65.

33. ZIMMER L, DELION-VANCASSEL S, DURAND G, GUILLOTEAU D, BODARD S, BESNARD JC, CHALON S (2000). Modification of dopamine neurotransmission in the nucleus accumbens of rats deficient in $\mathrm{n}$ 3 polyunsaturated fatty acids. J Lipid Res, $41: 32-40$. 
34. WAINWRIGHT P (1992). Do essential fatty acids play a role in brain and behavioral development ? Neurosci Behav Rev, 16 : 193-205.

35. FRANCES H, MONIER C, BOURRE JM (1995). Effects of dietary alpha-linolenic acid deficiency on neuromuscular and cognitive function in mice. Life Sci, 57 : 1935-47.

Illustrations

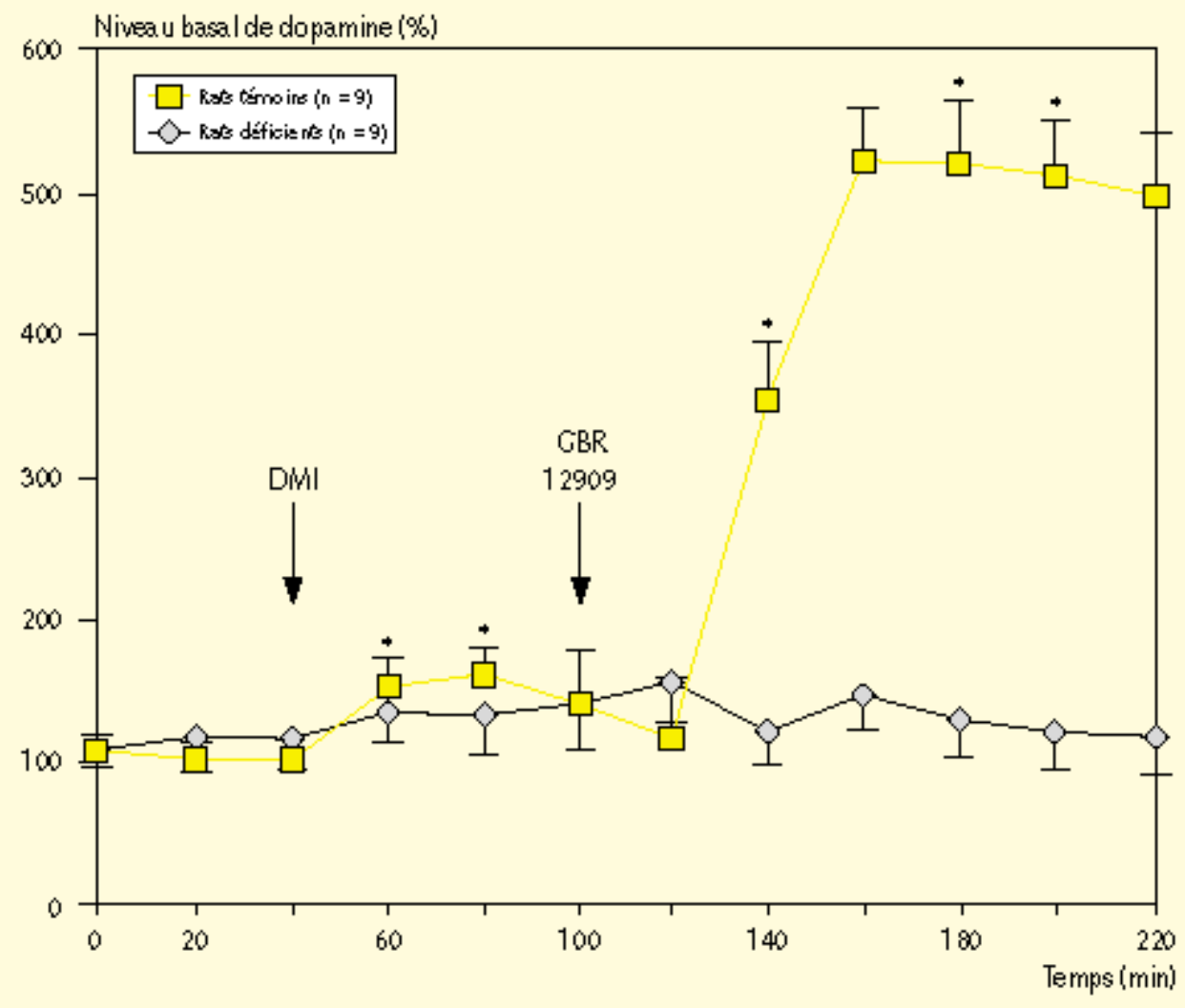

Figure 1. Étude de microdialyse intracérébrale - cortex frontal.

Libération de dopamine sous stimulation à la désipramine (DMI) et au GBR 12909, chez des rats déficients en AGPI $n-3$ comparés à des animaux recevant un régime équilibré ; ${ }^{*}: p<0,05$ entre les deux groupes alimentaires (d'après Zimmer et al. [32]). 

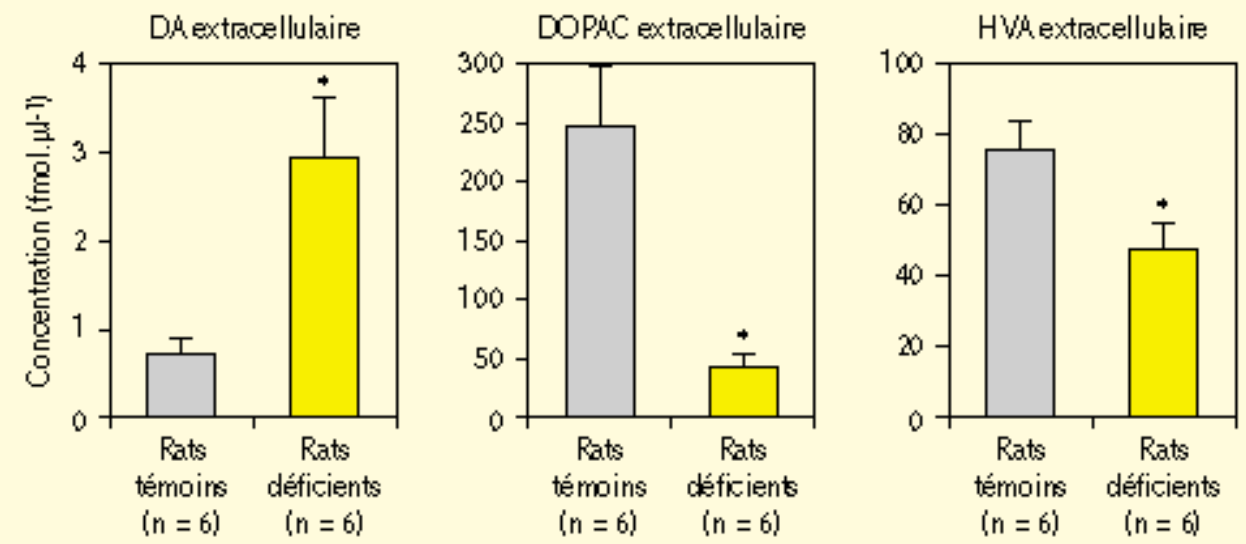

Figure 2. Étude de microdialyse intracérébrale - noyau accumbens.

Libération basale de dopamine (DA) et de ses principaux métabolites (Dopac et HVA)

chez des rats déficients en AGPI n-3 comparés à des animaux recevant un régime

équilibré ; ${ }^{*}: p<0,05$ entre les deux groupes alimentaires (d'après Zimmer et al. [33]). 

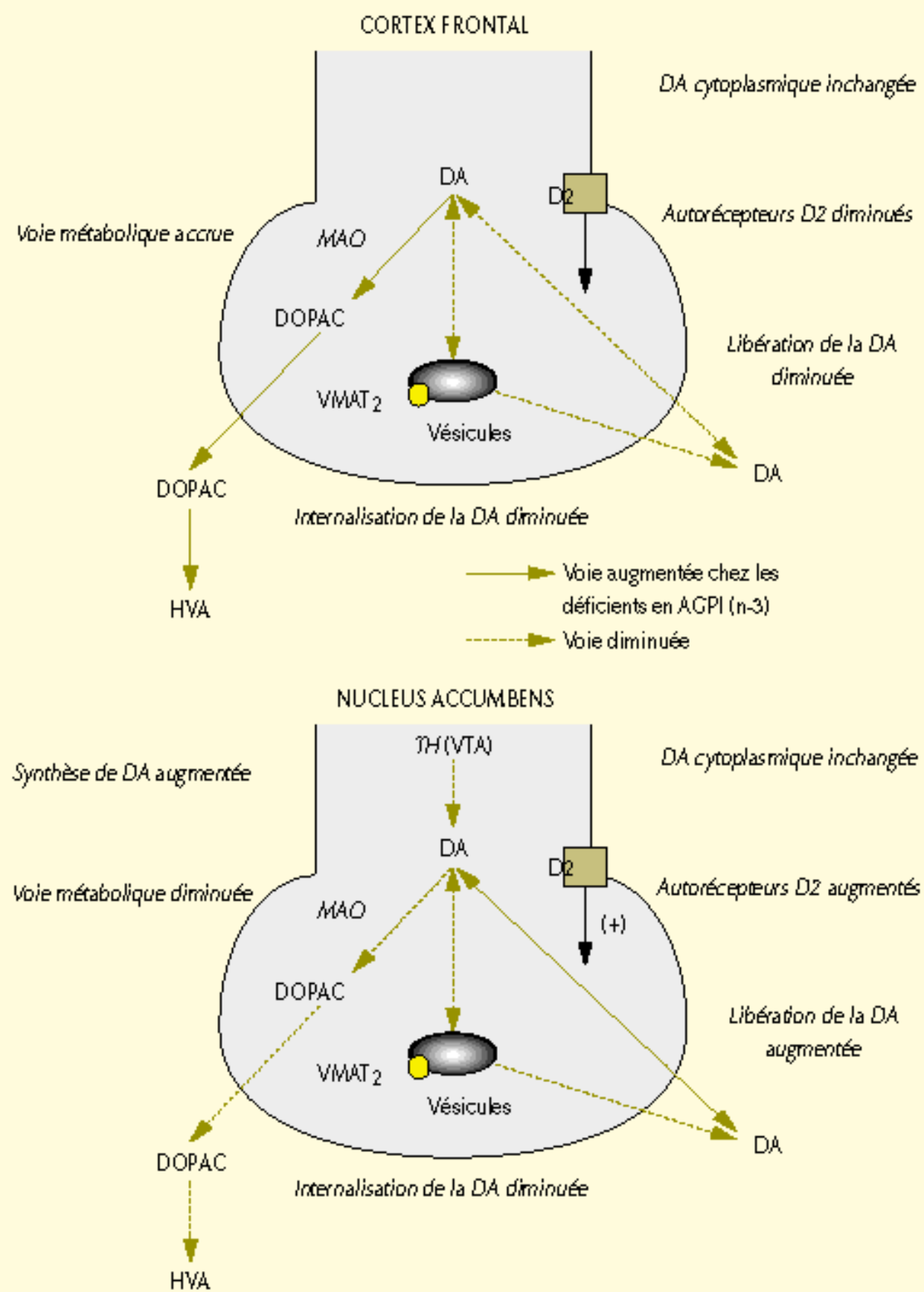

Figure 3. Mécanisme pouvant rendre compte des anomalies de la neurotransmission dopaminergique induites dans le cortex frontal et le noyau accumbens par la déficience en AGPI $n-3$. 


\begin{tabular}{|c|c|c|c|}
\hline & \multirow[b]{2}{*}{$\mathrm{n}$} & \multicolumn{2}{|c|}{$\begin{array}{c}\text { AC totaux } \\
\%\end{array}$} \\
\hline & & $\Sigma_{\omega_{3}}$ & $\Sigma_{u_{16}}$ \\
\hline Nigériens & 38 & $13,4 \pm 0,70$ & $30,3 \pm 0,78$ \\
\hline Suédois (nord) & 17 & $13,1 \pm 1,36$ & $35,8 \pm 1,06$ \\
\hline Suédois (sud) & 19 & $8,68 \pm 0,73$ & $37,4 \pm 0,98$ \\
\hline Australiens & 33 & $7,35 \pm 0,33$ & $39,9 \pm 0,53$ \\
\hline Minnesota (omnivores) & 100 & $5,53 \pm 0,13$ & $42,1 \pm 0,23$ \\
\hline Minnesota (végétariens) & 23 & $5,48 \pm 0,31$ & $41,0 \pm 0,42$ \\
\hline Bulgares & 30 & $5,26 \pm 0,42$ & $39,0 \pm 0,46$ \\
\hline Australiens aborigènes & 93 & $4,69 \pm 0,12$ & $37,7 \pm 0,36$ \\
\hline Australiens enfants & 48 & $4,78 \pm 0,25$ & $34,9 \pm 0,98$ \\
\hline Américains enfants & 38 & $3,57 \pm 0,19$ & $38,1 \pm 0,68$ \\
\hline
\end{tabular}

Tableau 1. Statut en AGPI n-3 et AGPI n-6 des phospholipides plasmatiques dans différentes populations (d'après Holman [1]).

\begin{tabular}{|lccc|}
\hline & & \multicolumn{2}{c}{$\begin{array}{c}\text { Valeurs contrôles } \\
\% \\
\text { (Minnesota omn ivores) }\end{array}$} \\
\cline { 2 - 4 } & & \multicolumn{2}{c}{$\boldsymbol{\Sigma}_{\boldsymbol{\omega}_{\mathbf{3}}}$} \\
\hline Anorexie nerveuse & $\mathbf{n}$ & 71 & 86 \\
Ma adie de Huntington & 28 & 63 & 84 \\
Sclérose multiple & 14 & 76 & 90 \\
Rétinite pigmentaire & 10 & 68 & 90 \\
\hline
\end{tabular}

Tableau 2. Statut en AGPI n-3 et AGPI n-6 des phospholipides plasmatiques dans différentes maladies du système nerveux central (d'après Holman [1]). 


\begin{tabular}{|lccc}
\hline \multirow{2}{*}{ AGPI } & \multicolumn{3}{c}{$\mathrm{mg} / 100 \mathrm{mg}$ phospholipides } \\
\cline { 2 - 4 } & $\begin{array}{c}\text { Témoins } \\
(\mathrm{n}=16)\end{array}$ & $\begin{array}{c}\text { Schizoph rén ie } \\
(\mathrm{n}=23)\end{array}$ & $\mathbf{p}$ \\
\hline $18: 2 \mathrm{n}-6$ & $14,2 \pm 2,0$ & $9,7 \pm 0,6$ & $<0,001$ \\
$20: 4 n-6$ & $17,3 \pm 3,6$ & $11,9 \pm 6,2$ & $<0,002$ \\
$20: 5 n-3$ & $1,6 \pm 0,4$ & $1,0 \pm 0,4$ & $<0,001$ \\
$22: 6 n-3$ & $7,2 \pm 1,3$ & $3,7 \pm 2,4$ & $<0,001$ \\
\hline
\end{tabular}

Tableau 3. Composition en AGPI des phospholipides érythrocytaires - Schizophrénie (d'après Peet et al. [8]).

\begin{tabular}{|lccc}
\hline \multirow{2}{*}{ AGPI } & \multicolumn{3}{c}{$\begin{array}{c}\text { AG totaux } \\
\%\end{array}$} \\
\cline { 2 - 4 } & $\begin{array}{c}\text { Témoins } \\
(\mathrm{n}=15)\end{array}$ & $\begin{array}{c}\text { Dépression } \\
(\mathrm{n}=15)\end{array}$ & $\mathbf{p}$ \\
\hline $18: 2 \mathrm{n}-6$ & $10,54 \pm 1,30$ & $8,45 \pm 2,32$ & $<0,005$ \\
$18: 3 \mathrm{n}-3$ & $0,24 \pm 0,16$ & $0,13 \pm 0,17$ & $<0,06$ \\
$20: 4 \mathrm{n}-6$ & $14,2 \pm 3,2$ & $11,4 \pm 4,4$ & $<0,06$ \\
$22: 5 \mathrm{n}-3$ & $2,50 \pm 1,07$ & $1,61 \pm 1,21$ & $<0,04$ \\
$22: 6 \mathrm{n}-3$ & $5,43 \pm 2,01$ & $3,11 \pm 2,47$ & $<0,009$ \\
$\Sigma \mathrm{n}-6$ & $32,3 \pm 4,8$ & $26,3 \pm 8,0$ & $<0,02$ \\
$\Sigma \mathrm{n}-3$ & $9,04 \pm 3,40$ & $5,39 \pm 4,16$ & $<0,3$ \\
$\mathrm{n}-6 / \mathrm{n}-3$ & $3,55 \pm 0,89$ & $3,90 \pm 0,85$ & $<0,06$ \\
AAVHA & $2,61 \pm 0,59$ & $3,23 \pm 0,91$ & \\
& & & \\
\hline
\end{tabular}

Tableau 4. Composition en AGPI des phospholipides érythrocytaires - Dépression (d'après Peet et al. [17]). 


\begin{tabular}{|c|c|c|c|}
\hline \multirow[b]{2}{*}{ AGPI } & \multicolumn{3}{|c|}{$\begin{array}{c}\text { AG totaux } \\
\%\end{array}$} \\
\hline & $\begin{array}{l}\text { Témoins } \\
(n=35)\end{array}$ & $\begin{array}{l}\text { ADHD } \\
(n=46)\end{array}$ & $\mathbf{p}$ \\
\hline $18: 2 n-6$ & $9,70 \pm 1,54$ & $9,28 \pm 2,24$ & ns \\
\hline $18: 3 n-3$ & - & - & - \\
\hline $20: 4 n-6$ & $15,12 \pm 2,39$ & $13,74 \pm 2,75$ & $<0,02$ \\
\hline $22: 5 n-3$ & $1,54 \pm 1,35$ & $1,58 \pm 1,51$ & ns \\
\hline $22: 6 n-3$ & $2,18 \pm 1,45$ & $1,61 \pm 1,31$ & $<0,06$ \\
\hline$\Sigma n-6$ & $32,05 \pm 4,46$ & $30,26 \pm 5,38$ & ns \\
\hline$\Sigma \mathrm{n}-3$ & $3,72 \pm 2,77$ & $2,95 \pm 2,59$ & ns \\
\hline$n-6 / n-3$ & $8,01 \pm 3,47$ & $10,63 \pm 6,36$ & ns \\
\hline
\end{tabular}

Tableau 5. Composition en AGPI des phospholipides érythrocytaires - Attention

Deficit/Hyperactivity Disorder (d'après Stevens et al. [22]). 